\title{
Violence et espace urbain
}

Un axe de recherche du réseau Saisir l'Europe

Ariane Jossin et Teresa Koloma Beck

\section{(2) OpenEdition}

Journals

Édition électronique

URL : http://journals.openedition.org/ifha/7409

DOI : 10.4000/ifha.7409

ISSN : 2198-8943

Éditeur

IFRA - Institut franco-allemand (sciences historiques et sociales)

Édition imprimée

Date de publication : 1 décembre 2013

ISSN : 2190-0078

Référence électronique

Ariane Jossin et Teresa Koloma Beck, « Violence et espace urbain », Revue de l'IFHA [En ligne], 5 | 2013,

mis en ligne le 17 février 2014, consulté le 23 avril 2019. URL : http://journals.openedition.org/

ifha/7409 ; DOI : 10.4000/ifha.7409

Ce document a été généré automatiquement le 23 avril 2019

(CIFHA 


\title{
Violence et espace urbain
}

\author{
Un axe de recherche du réseau Saisir l'Europe
}

Ariane Jossin et Teresa Koloma Beck

\section{Introduction}

1 L'axe «Violences urbaines » se consacre à l'historicisation et à la problématisation d'un phénomène qui suscite un vaste intérêt dans un climat de protestations sociales, de terrorisme et d'éruptions de violence individuelle ou collective. Au moyen d'enquêtes historiques ou sociologiques, il vise à faire de la notion de « violences urbaines » un outil d'analyse efficace pour des études systématiques et empiriques. Cette notion n'implique pas de compréhension essentialiste de l'espace, mais se réfère au concept d'« espace social ", dont les structures sont appropriées, déterminées et fixées par des acteurs, des discours et des pratiques. Une attention particulière est accordée aux conditions historiques spécifiques de la construction des "espaces de violences urbaines", mais aussi à l'interaction complexe des expériences concrètes, du discours médiatique et des politiques de sécurité. Le projet abordera deux aspects de la dimension historique des " violences urbaines »: d'une part, la permanence du discours sur les violences urbaines, présent dès le Moyen Âge et la Renaissance comme élément constitutif de la description des interactions urbaines; d'autre part, l'ambivalence de la ville comme espace de violence et de protection, à étudier également dans une perspective de longue durée. Les questions traitées par le groupe de travail s'articuleront autour (1) de la construction et de la déconstruction des espaces de violences urbaines, (2) de l'analyse comparée des espaces de violences urbaines en Europe, (3) de la combinaison de la réalité des violences urbaines et de leur conversion en catégories d'analyse pour les sciences sociales.

2 L'axe «Violences urbaines » est divisé en deux grands thèmes : «Violences urbaines et logiques conflictuelles» et «Violences urbaines et logiques sociales». Deux projets de recherche post-doctoraux et quatre projets doctoraux sont actuellement consacrés à ces deux thématiques. La première partie de cet article présentera le premier de ces projets qui porte sur les phénomènes de violence urbaine dans des quartiers défavorisés à Paris et Berlin, au regard du genre et du rapport à l'espace. La deuxième partie s'attache au 
second projet qui porte sur les conflits globaux au sein de la ville. Ces deux analyses ont pour point de convergence leur intérêt pour le rôle de la violence dans la production d'espaces sociaux. Ils adoptent une approche comparatiste et spatialisée.

\section{Violence, genre et espace en quartiers défavorisés, à Paris et Berlin}

3 L'équipe française de l'axe "Violences urbaines» interroge l'usage qui est fait de la violence dans quatre quartiers défavorisés de Paris et Berlin. Il analyse également le rapport qu'ont les citoyens à la géographie de leur quartier, quand celui-ci est porteur d'une menace (réelle ou fantasmée) de violence. Enfin, il essaie de voir si ces phénomènes de violence et le rapport à l'espace de leur quartier répondent à un ordre genré. Cette démarche s'articule autour d'un refus de réduire le genre à l'opposition homme agresseur/femme victime pour saisir d'autres dynamiques de « violences » - par exemple, entre hommes avec des victimes masculines; ou à l'initiative de femmes dans le cadre de "bandes féminines »; ou encore sous la forme d'assignations géographiques (restriction de la liberté de se mouvoir) pour lesquelles il s'agira de voir si elles relèvent de la violence et/ou d'un genre donné.

4 D'un point de vue spatial, les études portant sur les phénomènes de violence donnent à voir deux formes de violences dont on livre volontiers une lecture genrée : une violence qui a lieu dans la rue et dont les acteurs sont des hommes, une autre qui se tient dans l'espace domestique et où la victime est le plus souvent une femme. ${ }^{1}$ Ce projet souhaite interroger ce sentiment d'une corrélation supposée entre genre et formes de violences en se demandant s'il est possible d'identifier un usage et un rapport à la violence qui relèveraient d'un genre déterminé. Nous proposons d'interroger ces représentations genrées associées à certaines formes de violence, à l'exemple de quatre quartiers défavorisés de Paris et Berlin:La Goutte d'Or, Clichy-Montfermeil, Nord-Neukölln, Gropiusstadt.

5 Les quartiers de la Goutte d'Or (Paris $18^{\mathrm{e}}$ ), Clichy-Montfermeil (Seine-Saint-Denis), NordNeukölln et Gropiusstadt (Berlin) ont été retenus selon deux critères principaux. 1/ Ces quartiers sont défavorisés et présentent un taux de pauvreté et de chômage nettement supérieurs à la moyenne des villes de leurs pays respectifs. Ils sont pour cette raison la cible de mesures publiques: une partie de la communauté d'agglomération de ClichyMontfermeil ( Grand ensemble, haut et bas ») est classée en Zone de Redynamisation Urbaine (ZRU, sous-catégorie des ZUS) ; le quartier de la Goutte d'Or compte depuis 1996 parmi les Zones urbaines sensibles (ZUS) et sa partie Nord (Château rouge) a été inscrite en septembre 2012 dans la première liste des Zones de Sécurité Prioritaires (ZSP); à Berlin, une partie importante des quartiers de Nord-Neukölln et de Gropiusstadt sont classés «Gebiet mit besonderem Entwicklungsbedarf " (littéralement "Territoire aux besoins en développement particuliers »). Nord-Neukölln a été par ailleurs classé parmi les cinq quartiers catégorisés «Aktionsräume Plus » par la ville de Berlin et fait ainsi l'objet d'une action urbaine et sociale plus appuyée. 2/ La Goutte d'Or et Neukölln sont des quartiers intra-muros ou centraux des deux capitales européennes, tandis que ClichyMontfermeil et Gropiusstadt sont situés en périphérie et présentent une architecture de type grands-ensembles. Le contraste de ces deux implantations urbaines permet 
d'interroger l'impact de la centralité et de la vétusté des lieux de vie sur le rapport à l'espace.

6 Le constat (non étayé, à ce stade du projet) d'une assignation géographique et d'une restriction de la liberté de mouvement de jeunes femmes dans ces quartiers a été le point de départ de la présente réflexion ${ }^{2}$. Nous nous sommes alors demandé si cette contrainte posée à la liberté de se mouvoir dans l'espace public pouvait être considérée comme une forme de violence "symbolique» ou psychologique ${ }^{3}$ - par distinction à la violence physique et directe. Ce constat a ensuite voulu être élargi au cas des hommes, en se demandant si, eux aussi, subissaient des formes d'assignations géographiques, moins visibles pour le chercheur sur le terrain. Le projet veut ainsi étudier le bannissement des femmes et/ou des hommes de certains lieux qui prend différentes formes : par exemple, l'interdiction pour un homme de fréquenter des activités de loisirs réservées aux femmes, ou l'impossibilité pour une femme de prendre un verre dans un bar de son quartier, jusqu'à la séquestration d'une femme par son mari. L'apposition du qualificatif psychologique au terme de violence soulève la question de la conversion des assignations observées (de manière exploratoire) en catégorie d'analyse des sciences sociales, et plus généralement celle de la définition donnée à la « violence ». Elle nécessite de se pencher sur les éventuelles conséquences (atteintes aux personnes, harcèlement, agressions) que peuvent avoir la transgression des assignations spatiales, les passages de « frontières » ou les transgressions de normes.

7 Au-delà de cette approche en termes de spatialité et de territorialité, le projet étudiera plus largement les phénomènes de violence (notamment la violence intrafamiliale, la délinquance, les altercations avec la police) au prisme du genre dans ces quatre quartiers défavorisés de Paris et Berlin. Loin de proposer une typologie figée, il s'agira de voir dans quelle mesure les différents types de violence identifiés sont souples et s'imbriquent de manière synchronique (par exemple, les « guerres de bandes » s'accompagnent de conflits avec la police) ou diachronique (étude de la corrélation entre violences subies dans l'enfance et des comportements sociaux violents).

8 Nos analyses reposeront sur 80 entretiens semi-directifs et sur des "cartes mentales " effectués en 2013-2014. En attendant de pouvoir livrer des résultats propres à notre projet, cet article présente quelques hypothèses et pistes d'analyse à partir du dépouillement exploratoire de données récoltées en 2008-2011, qui nous renseignent sur le rapport à l'espace et les rapports sociaux de sexe dans les mêmes quatre quartiers. Elles ont été collectées dans le cadre du projet ANR-DFG «Stratégies professionnelles et passage à l'âge d'adulte de jeunes descendants d'immigrés en France et en Allemagne ", coordonné conjointement avec Carsten Keller (Université de Duisburg-Essen), Olaf GrohSamberg (Université de Bremen) et Ingrid Tucci (Deutsches Institut für Wirtschaftsforschung Berlin). Ce projet ANR-DFG portait exclusivement sur le cas de jeunes descendants d'immigrés dont les parents étaient issus du Maghreb, de l'Afrique subsaharienne, de Turquie ou du Proche-Orient. L'étude des assignations spatiales en quartiers défavorisés que nous projetons de mener ces trois prochaines années au sein du Réseau «Saisir l'Europe » ne justifie en rien que l'on restreigne l'échantillon d'étude aux descendants d'immigrés, ni que l'on retienne un critère d'âge. C'est pourquoi nous veillerons à interroger en 2013-2014 des personnes de tout âge, avec ou sans ascendance migratoire sur leur rapport à la violence au regard du genre et de l'espace, dans ces mêmes quatre quartiers. Le présent texte expose des extraits de données qui ont permis 
la construction d'hypothèses et d'une problématique qui seront développées au sein du réseau « Saisir l'Europe ».

\section{a) Les assignations spatiales rapportées par les jeunes femmes}

9 «Si tu veux être tranquille avec ta mère [le ton monte d'un cran], que tu ne lui donnes pas une raison de te niquer ta gueule le soir quand tu rentres, et ben, tu fermes ta gueule en classe, tu fais ta petite bouffonne, tu ramènes la moyenne. (...) Mais surtout une chose : ne te fais pas remarquer! Parce que tu vas te faire massacrer. Et on s'est tous fait taper à l'époque. Alors, ça peut être le balai, ça peut être le fil électrique, ça peut être des marques où tu ne vas pas à la piscine » (Femme, 32 ans, restauratrice, Paris).

10 Cette jeune femme interviewée en 2009 évoque tout le long de l'entretien que nous avons mené avec elle (autour de sa trajectoire biographique et professionnelle) les rapports sociaux de sexe qui animent et dominent sa famille et son quartier (Paris $18^{\mathrm{e}}$ ). Elle dénonce, d'une part, les rôles ou les comportements fortement codifiés et réglementés auxquels sont assignés les jeunes femmes : elle ne peut pas fumer dans son quartier, elle ne peut pas se faire raccompagner par son compagnon chez elle, elle a rencontré de nombreux obstacles venant des hommes de sa famille lorsqu'elle a ouvert son établissement de restauration, etc. D'autre part, elle reproduit et justifie dans son discours ces assignations. Ainsi, elle évoque à plusieurs reprises le recours aux services de son frère pour régler ses ennuis au moyen de la démonstration de la force, ce qui met à jour les rapports complexes qui sous-tendent les relations de genre.

11 À Berlin, d'autres femmes rapportent les assignations géographiques dont elles font l'objet au sein de leur quartier. Dans ces propos, il ressort que c'est généralement le risque d'une confrontation homme-femme qui constitue l'achoppement de la liberté de mouvement de ces femmes :

12 «Mon mari m'a aussi menacée avec une arme. (...) Un pistolet. (...) J'étais allée faire un babysitting et il devait venir me chercher (...) et il m'a dit "Je t'ai observée au $5^{\mathrm{e}}$ étage depuis la rue et tu as eu une affaire avec le père, tu as couché avec le père." Je me disais "Eh ?". Au bout d'un moment, je suis devenue assez blasée par rapport à ça. (...) C'étaient des journées difficiles. Je pleurais, je me sentais vraiment rabaissée quand j'entendais de tels propos » (Femme, 38 ans, éducatrice, Berlin).

13 «Pour les Allemands (de ma formation), les choses sont différentes. Ils sortent faire la fête et rentrent tard à la maison, ce qui est inenvisageable pour moi. (...) [Et sortir en boîte ?] Non. [Mes parents] ne m'y autoriseraient pas. Même si j'étais majeure [note : elle a 21 ans]. Mais bon, c'est aussi lié à la religion. On ne doit pas montrer ses charmes, on ne doit pas danser devant le regard des autres » (Femme, 21 ans, en formation d'infirmière, Berlin).

14 À la suite de Sami Zegnani, nous nous demanderons aussi quelle est la perception qu'ont les femmes de leur exclusion partielle de l'espace public extérieur: envient-elles les hommes pour cette plus grande liberté (supposée) de mouvement ou voient-elles au contraire dans les regroupements masculins dans la rue, l'expression d'une fainéantise et d'une prédisposition à la délinquance $?^{4}$ Certaines soulignent les effets collatéraux de l'assignation au domicile familial ou l'influence des ami(e)s :

15 « Ouais, [les garçons] galèrent un peu plus [dans leurs études], ouais. Bah en même temps, ici, les garçons... il y en a beaucoup qui traînent dehors, tout ça... (...) [Pour les filles] c'est 
mal vu aussi, de traîner dehors, et tout, pour une fille, machin, euh... (...) Et puis les parents aussi [pensent que ce n'est pas leur rôle], je pense. Ouais, parce que bon... tu vois ta fille, tout ça, dehors, "han, j'ai vu ta fille, dehors", bla bla bla, ça la fout mal, et tout. C'est un peu comme un village, ici. On te voit dehors, et tout, "han, j'ai vu ta fille" et tout, machin... "À telle heure, avec un mec", et tout... Ça le fait pas, hein. (...) Ah, moi j’traîne pas ici, hein ! » (Femme, 22 ans, diplômée en hôtellerie et tourisme).

De même, Hughes Lagrange évoque une sociabilité familiale et féminine (comme compensation à un accès limité à l'espace public) qui peut offrir des libertés « restreintes mais protégées " pour les jeunes filles. ${ }^{5}$ Ainsi, nous interrogerons, dans un premier temps, les formes de domination qui sous-tendent le contrôle de ces jeunes femmes, tenterons d'identifier leurs auteur(e)s et nous poserons la question de la qualification de ces assignations : peut-on les qualifier de violence structurelle propre au quartier, ou encore de violence symbolique ou psychologique? La transgression de ces règles est-elle suivie de violence physique?

La possibilité d'utiliser à cet endroit certains outils de la sociologie de l'enfermement - tel le concept d'adaptations secondaires (secondary adjustments) d'E. Goffman ${ }^{6}$, comme moyen de se réapproprier sa vie en milieu confiné - sera envisagé. De la même manière, on pourra aborder ces stratégies de contournement à la lumière du concept de déviance ${ }^{7}$ : en quoi est-ce que la transgression de normes instituées (assignations géographiques par exemple) participe-t-elle de la construction de l'identité de ces femmes, entendue comme le fruit d'une négociation permanente entre actes d'attribution et principes d'identification? Enfin, si ces formes d'assignations spatiales doivent être lues en termes de limitation des libertés, on ne doit pas pour autant faire l'économie de se poser la question d'éventuels « effets positifs » de ces assignations, qui pourraient expliquer dans certains cas une éventuelle absence ou faiblesse de résistance. Tout d'abord, le sentiment d'illégitimité éprouvé par certaines femmes dans l'espace public extérieur (qui participe ainsi à la "reproduction sociosexuée » de l'espace) éclaire probablement en partie leur faible représentation dans les statistiques d'agressions dans l'espace public. ${ }^{8}$ Ensuite, le contrôle parental plus strict des jeunes filles pourrait être à l'origine, au moins en partie, de leur large sous-représentation parmi les jeunes en décrochage scolaire. ${ }^{9}$

Enfin, notre volonté d'interroger le statut de "victime de la violence masculine" conférée aux femmes nous amènera à interroger le cas des «bandes de filles » qui nous renseignera sur le recours des jeunes femmes à des répertoires d'action ou des registres discursifs violents..$^{10}$ Ceci permettra de questionner la validité du prisme dominant d'une violence quasi exclusivement masculine.

\section{b) La violence et les rapports à l'espace dans les parcours sociobiographiques des hommes}

19 Si nos observations concernant les assignations spatiales tendent à conférer aux femmes interrogées un statut de victime, il est important de relever que les hommes sont aussi victimes d'une forme de "virilité " imposée par une frange d'hommes issus de ces quartiers ${ }^{11}$ : indirectement, parce qu'ils subissent les assignations de leurs sœurs, de leurs amies, de leurs compagnes, ou directement lorsque eux-mêmes ne peuvent investir des rôles conférés aux femmes (dans leur relation conjugale ou de père, notamment). Plus encore, on peut aller jusqu'à affirmer que les personnes à l'origine de ces préceptes sociaux genrés sont possiblement eux-mêmes pris au piège de leur système ${ }^{12}$, dans des 
quartiers qui sont à la fois un cocon et une prison, en ceci qu'ils protègent et enferment simultanément. ${ }^{13}$ D'autres assignations spatiales mériteront d'être interrogées, autour de la fréquentation de lieux de loisirs ou de consommation: discrimination à l'entrée des boîtes de nuit pour les descendants d'immigrés notamment ${ }^{14}$, ou impossibilité de s'asseoir dans un bar lorsqu'il est investi par les " grands frères ». ${ }^{15}$

Au-delà des violences intrafamiliales, de la délinquance et des assignations spatiales, nous étudierons également les phénomènes de violence liés aux rivalités entre bandes ${ }^{16}$; objet qui s'avère intéressant pour la cartographie des quartiers que nous planifions de concevoir. En effet, ces dynamiques de « cliques » répondent souvent à une territorialité bien définie. Enfin, la violence engendrée par les confrontations avec la police constitue un pendant de cette violence, guidée par des rivalités de bandes, par une volonté d'en découdre ou encore par des considérations commerciales (deal, recel, vente illégale). Celle-ci peut trouver son origine dans la répression d'une délinquance bien réelle, mais repose également, dans de nombreux cas, sur des contrôles de police répétés et non motivés par un délit. Ces contrôles constituent au moins une violence psychologique ou symbolique, sinon une violence physique quand ils « dérapent». On sait les concernant que les différences de traitement par les policiers jouent à la défaveur des populations descendantes d'immigrées ${ }^{17}$, plus particulièrement dans certains quartiers. Fabien Jobard a également montré que la probabilité d'être contrôlé était beaucoup plus élevée pour les hommes que pour les femmes, ainsi que pour les jeunes adultes. ${ }^{18}$ D'autre part, les attitudes discriminatoires émanant de la police sont très nettement plus fréquentes en France qu'en Allemagne ${ }^{19}$, ce qui renforce l'intérêt de notre approche comparatiste. Ce risque que peut comporter la rencontre d'une brigade de police devra être analysé au prisme du rapport à l'espace : quelles sont les stratégies d'évitement et les assignations spatiales liées à ces patrouilles? Quelle influence a eu, par exemple, l'implantation d'un commissariat à Clichy-sous-Bois en 2010 sur la mobilité des jeunes hommes et femmes ? Est-ce qu'au contraire, la confrontation à la police et donc la mise en situation de danger (où il est possible que le corps des interpelés souffre) peuvent être utilisées par les acteurs pour rendre visibles leur malaise social et leur souffrance, en désignant l'État comme responsable de leurs corps souffrants ?20

\section{Conflits globaux dans la ville}

21 Le deuxième projet de l'axe «Violences urbaines" s'intitule «Conflits globaux dans la ville » et interroge le rôle des villes dans les dynamiques de conflits globalisés. Ce ne sont pas des phénomènes de violence propres à la ville même qui en constituent le cœur ; l'intérêt porte davantage sur la ville comme théâtre d'importants conflits à l'échelle sociale mondiale. Ce projet considère les villes comme des lieux au sein d'une «société mondiale » (Weltgesellschaft) et comme des points de cristallisation de dynamiques de la globalisation. Parmi ces derniers ne figurent pas seulement les processus croissants d'intégration économique, politique et culturelle, mais aussi des conflits qui sont réglementés au niveau de cette société mondiale. Sur fond d'interconnexions médiatiques, économiques, sociales et politiques (sécuritaires) accrues, la défrontalisation est un des signes caractéristiques de ces conflits. Ainsi, la « défrontalisation » signifie ici bien plus qu'un simple "dépassement des frontières": ce concept renvoie à la multiplication des références spatiales significatives pour l'un ou l'autre conflit. $\mathrm{Au}$ regard de cette multiréférentialité, ces conflits ne peuvent plus être envisagés autour 
d'un seul lieu : ils ne sont plus locaux, nationaux ou encore régionaux mais plurilocaux ${ }^{21}$. Un épisode clé22 à cet égard s'est produit avec les attaques du 11 septembre 2001 et le War on Terror qui a suivi.

Parmi les exemples plus récents figurent les attentats d'Oslo et Utøya 2012, les actes de violences et attentats consécutifs à la sortie du film sur Mahomet intitulé «L'innocence des musulmans » (fin 2012) ou bien aussi les protestations qui dégénèrent régulièrement à l'occasion des rencontres du G8/G20. Les acteurs et observateurs accordent une importance au niveau mondial à chacun de ces événements, qui sont à l'origine ancrés dans un espace urbain spécifique. En contraste avec ce que certains discours populaires suggèrent, de tels conflits ne freinent pas la globalisation, mais, au contraire, accélèrent la production de structures globalisées. On pense enfin à la formation de sujets qui se comprennent eux-mêmes dans l'horizon du monde.

Les conflits ainsi définis mettent la recherche face à des défis singuliers, aussi bien au niveau des concepts théoriques que des outils méthodologiques. En effet, ils rompent avec les distinctions qui étaient, et sont encore, structurantes pour l'étude des conflits au sens le plus large : oppositions entre conflits internes à l'État et conflits entre États, conflits sociaux et conflits politiques, acteurs étatiques et non-étatiques. Ces distinctions perdent de leur efficacité analytique puisqu'elles prennent l'État-Nation comme principale référence spatiale. Les conflits du présent ont néanmoins lieu dans les conditions de la globalité, entendue comme la pensée et l'agir d'individus et de collectifs «dans l'horizon du monde $\aleph^{23} \mathrm{Au}$ vu de cette globalité, la signification des structures nationales étatiques comme modèle d'ordre dominant au plan global s'amenuise. Cela est dû à une démultiplication de références qui concerne également la production de conflit. Les conflits deviennent - comme dans le cas du War on Terror ou de la protestation contre le film sur Mahomet - plurilocaux; en d'autres termes, ils sont négociés simultanément dans des arènes diverses mais interdépendantes, qui sont ancrées différemment dans l'espace. Les conditions d'émergence et les dynamiques de (re)production de ces conflits plurilocaux sont jusqu'à présent peu comprises du point de vue théorique et peu explorées de manière empirique ${ }^{24}$. Ce deuxième projet de l'axe "Violences urbaines " viendra combler cet écueil.

\section{a) Focus : les villes comme points de cristallisation des dynamiques de conflits}

En tant que points de cristallisation des processus de globalisation, les villes acquièrent une importance particulière pour définir les «dynamiques de conflits». Elles représentent des systèmes sociaux dans lesquels les interdépendances médiatiques, économiques, sociales et politiques (sécuritaires) caractéristiques de la globalisation, se matérialisent en se concentrant en un lieu concret. À cet égard, Hartmut Häußermann soutient que la ville en sciences sociales ne peut jamais être comprise uniquement à partir d'une perspective locale, mais que son analyse doit constamment s'effectuer sur plusieurs niveaux et prendre notamment en considération le global ou le transnational. ${ }^{25}$ Ainsi les villes deviennent les foyers de conflits plurilocaux. Des dynamiques distinctes, en partie opposées, sont en jeu : il faudra s'intéresser en premier lieu, à l'hétérogénéité sociale, culturelle, religieuse et économique propre aux villes tout comme aux relations sociales qui créent les conditions pour la (re)production et l'escalade des conflits, y compris au potentiel de violence qui y est lié. Deuxièmement, en tant que lieux porteurs 
de symboles, les villes constituent des scènes idéales pour une entrée en jeu efficace de la dissension, de la protestation ou de la résistance; en effet dans l'espace public des villes se concentrent des lieux symboliques (églises, banques, bâtiments gouvernementaux, etc.). Ainsi une protestation dans un endroit donné peut évoluer vers une offensive contre un système tout entier. En troisième lieu, les villes et leur concentration de capital social, politique et culturel ne renferment pas seulement des structures favorisant l'escalade de la violence, mais aussi des organismes visant à transformer les structures de conflits et à pacifier la violence, comme des autorités et ministères en matière d'organisations internationales ou des organisations non gouvernementales (ONG).

En amont de ces réflexions, le projet situe la ville comme point de départ de l'observation de conflits globalisés. Il pose la question des conditions d'émergence, des dynamiques de reproduction et de transformation des conflits violents plurilocaux et fait en ce sens de l'espace urbain un champ crucial d'investigation. Il ne s'agit pas ici de savoir dans quelle mesure les flux mondiaux de biens et de personnes peuvent être à l'origine de la violence urbaine, mais avant tout de s'interroger sur la manière dont des événements violents déterminés - comme les attentats du 11 septembre 2001 et le War on Terror consécutif produisent de la globalité, et avant tout des phénomènes sociaux. Primo, une attention particulière est consacrée aux processus de construction sociale de la plurilocalité, à la manière dont interdépendances et interactions sont établies à la fois dans l'action et dans la perception des acteurs à différents niveaux - local, global, national, etc. - et en différents lieux. Secundo, dans ce cadre, un intérêt particulier concerne le rôle joué par l'entrée en jeu de la violence dans ces dynamiques. En effet, la violence produit d'une part toujours de la localité, car elle touche des corps ou autres structures physiques. D'autre part, les phénomènes violents font rapidement naître des rapports translocaux, car ils attirent l'attention d'observateurs et en fonction de la symbolique du lieu, ils peuvent par leur signification largement dépasser le lieu où ils se produisent. De tels épisodes de violence ne concernent alors plus seulement les structures de la ville, mais celles de la société mondiale et le cas échéant, des États. Cela s'explique par l'institutionnalisation de critères d'observation, de normes et de valeurs universalistes qui est une composante des processus modernes de globalisation, tout particulièrement de l'établissement de la nonviolence comme norme globale. Cette évolution n’a évidemment pas entraîné la disparition des moyens de coercition physique en tant que stratégie de conflit. Elle a tout de même transformé les conditions de reproduction des conflits violents. En effet, la norme de la non-violence universelle gagnant en validité, la violence même devient un scandale qui éveille l'attention d'un public global. La norme universaliste permet ainsi_de faire d'un conflit où la violence est observée un objet non seulement de la politique internationale mais également des processus globaux de communication. ${ }^{26} \mathrm{Tertio}$, on doit envisager le type de répercussions engendrées pour la ville elle-même à partir du rôle de celle-ci comme lieu de production et de reproduction des conflits violents globalisés.

Le projet creuse cette interrogation dans le cadre d'une étude sociale de type qualitatifempirique dont l'objet central est le War on Terror et son camp adverse, le «Djihad global ». Le noyau de la recherche est une ethnographie pluri-site (multi-sited ethnography ). Cette dernière est une méthode de recherche développée depuis le milieu des années 1990 en anthropologie qui a pour but de faciliter la recherche ethnographique à l'ère de la globalisation. Alors que la recherche anthropologique était traditionnellement liée à un terrain unique, l'ethnographie pluri-site ne place pas le terrain au centre mais se penche sur un phénomène social dans plusieurs espaces. ${ }^{27}$ Dans le projet de recherche ici exposé, 
il s'agit du conflit qui se développa au lendemain des attentats du 11 septembre 2001, et qui est communément perçu comme une confrontation entre l'islam radical et les valeurs occidentales. Ce conflit semble particulièrement adapté pour la problématique, c'est-àdire la prolifération des lieux où on le négocie. Il touche aujourd'hui tous les États et toutes les régions où les populations musulmanes sont nombreuses. Ainsi le War on Terror/ Global Djihad est aujourd'hui mené aux États-Unis exactement de la même manière qu'en Inde, au Nigéria ou en Allemagne. Pour des raisons pragmatiques cette recherche choisit l'Allemagne comme point de départ.

\section{b) Approche : Production de globalité dans les conflits violents}

Par sa problématique, le projet œuvre au point d'intersection entre recherche sur la globalisation, recherche sur le conflit et étude sur la ville. Ses objectifs se trouvent sur deux plans: d'une part, les conditions et dynamiques de (re)production des conflits violents plurilocaux ainsi que leurs répercussions sur les espaces urbains doivent être explorées et reconstruites. D'autre part, il s'agit d'aller au-delà et d'apporter une contribution aux termes théoriques et conceptuels sur de tels conflits et phénomènes de violence.

Le lien entre globalisation, urbanité et conflit violent a été jusqu'à présent peu exploré. Même si la recherche récente sur la globalisation étudie intensément le rôle des villes dans le processus de mondialisation, elle est centrée sur la fonction des métropoles comme plaque tournante des flux financiers, économiques et de l'information ${ }^{28}$. Dans cette perspective les conflits, et plus particulièrement les plus violents, apparaissent comme des incidents liés aux dynamiques de l'intégration globale. Le présent projet opte pour un point de départ alternatif: dans la lignée des pionniers de la sociologie des conflits, Georg Simmel et Lewis Coser, les conflits sont appréhendés comme des processus de formation de la structure sociale. Dans sa sociologie apparue pour la première fois en $1908^{29}$, Simmel présente le conflit (qu'il qualifie de « $\mathrm{Kampf}^{30}$ » dans sa terminologie) comme une des formes cardinales de la socialisation. Il écrit : «Si toute interaction parmi les hommes est une socialisation, le conflit qui est bien une des interactions les plus vives (...) doit alors être pleinement considéré comme une socialisation $»{ }^{31}$ Les conflits apparaissent par conséquent comme des processus sociaux qui érigent et stabilisent des structures sociales par le biais de la négociation. ${ }^{32}$ Après sa réception par Coser aux ÉtatsUnis, le travail simmelien gagne en influence dans le domaine des sciences sociales. Dans l'ouvrage paru en 1956, The Functions of Social Conflict, le sociologue affûte les propositions simmeliennes; c'est la naissance de la première théorie sociale du conflit, ce dernier n'étant plus reconstruit comme producteur ou symptôme de désintégration sociale mais, au sens fonctionnel, comme processus productif qui produit ou transforme les structures sociales. ${ }^{33}$

29 La recherche associe cette conception du conflit comme événement producteur de social avec un modèle de globalisation dont l'attention ne porte pas sur l'intégration économique ou politique progressive, mais sur les processus de convergence entre horizons de représentation et horizons d'attentes. Les théories de la globalisation, propres à la théorie de la communication, jouent un rôle moteur en mettant avant tout en relation le phénomène avec l'émergence d'une opinion publique mondiale ou encore d'un "public global» qui se constitue en communiquant autour de valeurs universalistes institutionnalisées, comme par exemple les droits de l'homme. Des observateurs 
spécialisés, tels que journalistes, économistes et ONG, analysent les évènements dans le monde à l'aune de la conservation ou de la transgression des valeurs universelles. ${ }^{34}$ L'inclusion de cette perspective permet d'accentuer la compréhension analytique de la relation entre les dynamiques sociales de la violence et celles de l'espace.

\section{Synthèse}

30 L'approche spatiale est transversale à l'axe de recherche "Violences urbaines ». Les deux projets de recherche qui le constituent portent sur une analyse d'espaces, associés de manière temporaire ou durable, à un « potentiel » de violence. Cette projection « espaceviolence » repose sur des faits passés et situés dans l'espace (émeutes, par exemple), des associations cognitives ou locatives (lien entre un mouvement politique et des modes d'action violents, peur de certains lieux publics en période de vague terroriste) ou sur la stigmatisation d'espaces dans certains médias ou discours politiques (notamment la couverture médiatique des «banlieues»). Ces projets permettront de comprendre comment des phénomènes de violence peuvent transformer des structures spatiales et sociales.

\section{NOTES}

1. Marylène Lieber, Genre, violences et espaces publics, Paris: Presses de Sciences Po, 2008, p. 18. Dans le cas des violences conjugales et sexuelles, ce sont les femmes qui sont le plus souvent victimes d'hommes (le plus souvent le conjoint ou l'ex-conjoint) (Préfecture de Police, Les violences conjugales, Rapport interne, Paris, 2002, cité par Marylène Lieber, op. cit., p. 43-44, 53 ; voir aussi Marie-Lys Pottier, Philippe Robert, Renée Zauberman, Victimation et insécurité en Île-deFrance. Les résultats de la première enquête, Guyancourt: CESDIP, 2001. Par ailleurs, on observe en général un décalage certain entre sécurités « subjective » et « objective », et notamment dans le cas des femmes: celles-ci se sentent plus exposées aux agressions dans l'espace public, alors qu'elles en sont souvent moins victimes que les hommes (voir par exemple Marylène Lieber, op.cit ., p. 34-35 et 58; Wesley G. Skogan, «Public policy and the fear of crime in large American cities », in : John Gardiner, Public law and public policy, New York : Praeger, 1977 ; Philippe Robert, L'insécurité en France, Paris : La Découverte, 2002. Marylène Lieber relève à ce sujet une «double invisibilité » des violences faites aux femmes: les violences conjugales sont bien souvent occultées et celles dans l'espace public et spécifiques aux femmes sont peu prises en compte par les politiques publiques (Marylène Lieber, « La double invisibilité des violences faites aux femmes dans les contrats locaux de sécurité français », Cahiers du Genre, n 35, 2003, p. 71-94 ; voir aussi Ailbhe Smyth, «Résistance féministe à la violence masculine contre les femmes. Quelles perspectives? ", Nouvelles questions féministes, n 2, 2002, p. 76).

2. L'observation que nous évoquons ici a été faite en marge d'une précédente enquête qui a nécessité une forte présence sur le terrain. Il s'agit du projet ANR-DFG «Stratégies professionnelles et passage à l'âge d'adulte des jeunes issus de l'immigration en France et en Allemagne ", 2008-2011. Cette étude n'avait pas porté sur les questions de violence, de rapports sociaux de sexe ou de rapport à l'espace. 
3. Ce terme de "violence psychologique" ne recouvre que très partiellement ce que nous voulons décrire. Nous le préférerons toutefois ici à celui de « violence symbolique » en raison de la confusion que ce dernier terme pourrait induire avec le concept de Pierre Bourdieu (Pierre Bourdieu, La domination masculine, Paris : Le Seuil, 1998).

4. Sami Zegnani, «Occupation de la rue et rapport de genre chez les jeunes des cités ", Lettre du Printemps, $\mathrm{n}^{\circ} 11,2005$, p. 4.

5. Hugues Lagrange, Le déni des cultures, Paris : Le Seuil, 2010, p. 208.

6. Erving Goffman, Asylums: Essays on the social situations of mental patients and other inmates, Oxford: Anchor, 1961.

7. Howard Becker, Outsiders. Etudes de sociologie de la déviance, Paris : Métailié, 1985 (1963).

8. Steven Balkin, "Victimization rates, safety and fear of crime », Social problems, $n^{\circ} 3$, février 1979.

9. Olaf Groh-Samberg, Ariane Jossin, Carsten Keller, Ingrid Tucci, «Biographische Lebensentwürfe von Jugendlichen mit Migrationshintergrund», in: Jürgen Mansel, Karsten Speck (dir.), Jugend und Arbeit. Empirische Bestandsaufnahme und Analysen, Weinheim: Juventa, 2012 ; Olaf Groh-Samberg, Ariane Jossin, Carsten Keller, Ingrid Tucci, « Drift und zweite Chance. Bildungs- und Berufsverläufe von Migrantennachkommen in Frankreich und Deutschland », in : Kölner Zeitschrift für Soziologie und Sozialpsychologie, $\mathrm{n}^{\circ}$ spécial 52, 2012. Voir aussi le décalage au niveau de l'entrée en classe de $6^{\mathrm{e}}$, à l'âge «normal » ou en avance des jeunes filles et garçons d'origine turque et africaine : hors ZUS/ZEP on compte $71,8 \%$ de filles et $65,4 \%$ de garçons; en ZEP/ZUS, $60,9 \%$ de filles et $46,3 \%$ de garçons. Ceci montre selon Hughes Lagrange une "polarisation sexuée des comportements » plus marquée dans les "zones sensibles " (Hughes Lagrange, op. cit., p. 202-203).

10. Frederic Milton Thrasher, The gang. A study of 1313 gangs in Chicago, Chicago : The University of Chicago Press, 1963 (1927); Stéphanie Rubi, "Des adolescentes délinquantes", Les Cahiers Dynamiques, $\mathrm{n}^{\circ} 46,2010$; Stéphanie Rubi, «Les "crapuleuses": Masculinisation des comportements ou application de la loi des plus fortes? », VEI Enjeux, n 128, 2002 ; Sybille Artz, Sex, Power, and the Violent School Girl, New York: Teachers College Press, 1999 (1998); Meda Chesney-Lind, John Hagerdon, Female gangs in America, Chicago : Lake view press, 1999 ; Pascal Duret, Les jeunes et l'identité masculine, Presses Universitaires de France, Paris, 1999 ; Luce Michel, Adolescentes et violentes, Paris : Editions Michalon, 1999; Yazid Kherfi, Véronique Le Goaziou, Repris de justesse, Paris : Syros, 2000.

11. Boris Gobille, "Genre et mobilisations ", in : Sophie Béroud, Boris Gobille, Abdellali Hajjat et allii (dir.), Engagements, rebellions et genre dans les quartiers populaires en Europe, Paris: Archives contemporaines, 2011, p. 62.

12. Pierre Bourdieu, La domination masculine, Paris : Le Seuil, 1998, p. 75.

13. David Lepoutre, Cour de banlieue. Codes, rites et langages, Paris: Odile Jacob, 1997 ; Loïc Wacquant., "Les deux visages du ghetto. Construire un concept sociologique», Actes de la recherche en sciences sociales, $n^{\circ} 5,2005$, p. 12 ; Didier Lapeyronnie, Ghetto urbain, Robert Laffont, Paris, 2008 ; Michel Kokoreff, « Du stigmate au ghetto. De la difficulté à nommer les quartiers ", Informations sociales, $\mathrm{n}^{\circ} 141,2007$, p. 86-95 ; Michel Kokoreff., "Ghettos et marginalité urbaine », Revue française de sociologie, $\mathrm{n}^{\circ} 3,2009$, p. 553-572.

14. Lapeyronnie, op. cit., p. 510-513.

15. Éric Marlière, Jeunes en cité. Diversité des trajectoires ou destin commun?, Paris : L'Harmattan, 2005, p. 138-139.

16. François Dubet, Didier Lapeyronnie, Les quartiers d'exil, Paris: Le Seuil, 1992 ; Marwan Mohammed, "Les affrontements entre bandes : virilité, honneur et réputation ", Déviance et Société, $n^{\circ}$ 2, 2009; Marwan Mohammed, «La défiance des bandes: antagonismes sociaux et agressivité collective», Cités, n5 50, 2012; Marwan Mohammed, Laurent Mucchielli (dir.), Les bandes de jeunes. Des "blousons noirs" à nos jours, Paris : La Découverte, 2007. 
17. Fabien Jobard, "Police, justice et discriminations raciales", in : Didier Fassin, Eric Fassin (dir.), De la question sociale à la question raciale ?, Paris : La Découverte, 2006, p. 227.

18. Fabien Jobard, René Lévy, «Les contrôles d'identité à Paris ", Questions pénales, $n^{\circ} 1,2010$, p. 2. 19. Jérémie Gauthier, «Des corps étrange(r)s dans la police? Les policiers minoritaires à Paris et à Berlin ", Sociologie du Travail, $\mathrm{n}^{\circ}$ 4, 2011.

20. Voir à ce sujet les analyses en termes de «retournement de la violence contre soi » de Johanna Siméant, «La violence d'un répertoire : les sans-papiers en grève de la faim », Cultures \& Conflits, $\mathrm{n}^{\circ}$ 9-10, 1993.

21. Dans la recherche, ces conflits sont fréquemment accompagnés de l'attribut «transnational ». Ce concept ne sera délibérément pas utilisé ici car il est plus fortement lié au dépassement des frontières, au sens étroit de la défrontalisation.

22. Dernièrement les études sur la société mondiale (Weltgesellschaftsforschung) ont tenté de saisir de manière plus théorique les évènements mondiaux, qu'elles reconstruisent comme étant producteurs de la réalité sociale de la mondialisiation (Rudolf Stichweh, « Der 11. September 2001 und seine Folgen für die Entwicklung der Weltgesellschaft. Zur Genese des terroristischen Weltereignisses. ", in: Thorsten Bonacker, Christoph Weller (dir.), Konflikte der Weltgesellschaft. Akteure - Strukturen - Dynamiken, Frankfurt a. M. : Campus, 2006, p. 282-283). Voir aussi Stefan Nacke, René Unkelbach, Tobias Werron (dir.), Weltereignisse. Theoretische und empirische Perspektiven, Wiesbaden: VS Verlag, 2008, en particuler Rudolf Stichweh, «Zur Soziologie des Weltereignisses.», in: Stefan Nacke, René Unkelbach, Tobias Werron (dir.), Weltereignisse. Theoretische und empirische Perspektiven, Wiesbaden : VS Verlag, 2008.

23. Abdelwahab Meddeb, Benjamin Stora, «Arabisches Erwachen. Die Wiederaneignung des eigenen Schicksals in einer sich öffnenden Welt. (Von den Autoren durchgesehene Aufzeichnung eines Podiumsgesprächs während der États Généreaux du Renouveau am 29. Januar 2011 in Grenoble) ", Lettre Internationale, n 92, 2011, p. 23-25, p. 23.

24. La recherche récente sur les phénomènes de globalisation exige une nouvelle orientation fondamentale des sciences sociales, afin de saisir théoriquement les évolutions empiriques liées au processus de globalisation. Voir par exemple Philip Cerny, «Globalization and Other Stories : Paradigmatic Selection in International Politics. », in : Axel Hülsemeyer (dir.), Globalization in the 21st Century. Convergence and Divergence, London: Palgrave, 2003, p. 51; David Held, Anthony McGrew, «Introduction. Globalization at risk?», in: David Held, Anthony McGrew (dir.), Globalization theory. Approaches and controversies, Cambridge : Polity Press, 2007, p. 4-5 ; Katja Jung, Volk - Staat - (Welt-)Gesellschaft. Zur Konstruktion und Rekonstruktion von Kollektivitat in einer globalisierten Welt, Wiesbaden : VS Verlag, 2010, p. 89 ; Beate Kohler-Koch, « Einleitung. Effizienz und Demokrade. Probleme des Regierens in begrenzten Räumen.», Regieren in entgrenzten Räumen. Politische Vierteljahresschrift, nº spécial 29, 1998 ; Anthony McGrew, "Demokratie ohne Grenzen? Globalisierung und die demokratische Theorie und Politik.», in: Ulrich Beck (dir.), Politik der Globalisierung, Frankfurt a. M.: Suhrkamp, 1998; Richard Münch, «Politik in der globalisierten Moderne ", Soziale Welt, ${ }^{\circ}{ }^{\circ}$ spécial 14, 2003, p. 122 ; Susan Strange, The retreat of the state. The diffusion of power in the world economy, vol. 49, Cambridge : Cambridge University Press, 1996, p. 84; Spike Petersen, «Plural processes, patterned connections », Globalizations, n 1, 2004, p. 50; James Rosenau, «Many Globalizations. One International Relations », Globalizations, $\mathrm{n}^{\circ} 1$, 2004, p. 12; Martin Shaw, "Globality and historical sociology. State, revolution, and war revisited. ", in : Stephen Hobden, John M. Hobson (dir.), Historical sociology of International Relations , Cambridge : Cambridge University Press, 2002, p. 35.

25. Hartmut Häußermann, Walter Siebel, Stadtsoziologie eine Einführung, Frankfurt a. M. : Campus, 2004, p. 217.

26. Teresa Koloma Beck, Tobias Werron, «Gewaltwettbewerbe. 'Gewalt' in globalen Konkurrenzen um Aufmerksamkeit und Legitimität. », in: Stephan Stetter (dir.), Ordnung und Wandel in der Weltpolitik. Konturen einer Soziologie der Internationalen Beziehungen, Baden-Baden : 
Nomos, 2013. Voir aussi Teresa Koloma Beck, «The eye of the beholder. Violence as a social process ", International Journal of Conflict and Violence, $\mathrm{n}^{\circ} 2,2011$.

27. George Marcus, «Ethnography in/of the World System. The Emergence of Multi-Sited Ethnography ", Annual Review of Anthropology, n² 24, 1995, p. 95-117.

28. Voir en particulier à ce sujet le travail de Saskia Sassen, par exemple Saskia Sassen, The global city. New York, London, Tokyo, Princeton, New York: Princeton University Press, 1991; Saskia Sassen, Global networks, linked cities, New York: Routledge, 2002 ; Saskia Sassen, A sociology of globalization, New York : Norton, 2007.

29. Georg Simmel, Soziologie. Untersuchungen über die Formen der Vergesellschaftung, Frankfurt a. M. : Suhrkamp, 1992 (1908)..

30. Simmel distingue le "conflit» de la «concurrence » et range tous deux dans la catégorie générique de « différend» (Der Streit). La différence réside dans le fait que le conflit confronte directement les adversaires tandis que dans la concurrence, leur attention est focalisée sur un tiers, un public (voir aussi Tobias Werron, «Direkte Konflikte, indirekte Konkurrenzen. Unterscheidung und Vergleich zweier Formen des Kampfes », Zeitschrift für Soziologie, $\mathrm{n}^{\circ} 4,2010$, p. 302-18).

31. Georg Simmel, op. cit., p. 284.

32. ibid.

33. Lewis Coser, The functions of social conflict, Glencoe : Free Press, 1956.

34. Voir Bettina Heintz, Tobias Werron, «Wie ist Globalisierung möglich? Zur Entstehung globaler Vergleichshorizonte am Beispiel von Wissenschaft und Sport ", Kölner Zeitschrift für Soziologie und Sozialpsychologie, $\mathrm{n}^{\circ}$ 3, 2011.

\section{AUTEURS}

\section{ARIANE JOSSIN}

UMR IRICE, Paris Sorbonne

TERESA KOLOMA BECK

Centre Marc Bloch, Berlin 